\title{
High Pressure and moderate Temperature MEMS based Multi- function sensors
}

\author{
Baharul Islam², Saurav Zaman ${ }^{2}$ and J. Akhtar ${ }^{1}$ \\ ${ }^{1}$ Chief Scientist and Head, \\ Sensors \& Nano-Technology Group, Central Electronics Engineering Research Institute, Pilani-333 031, A \\ constituent Laboratory of CSIR, New Delhi, India \\ ${ }^{2}$ Assistant professor, Department of Electronics and communication Engineering, Assam down town University, \\ Guwahati, Assam
}

\begin{abstract}
A multifunction sensor based on bulk micromachining of silicon $\langle 100\rangle$ has been developed to measure dynamic change in pressure and temperature simultaneously. Boron doped polysilicon piezoresistors were placed on the silicon diaphragm in a full Wheatstone's bridge configuration employing standard microelectronics processes. Another single resistor has been placed on the chip but on the pressure non sensitive area of the chip for temperature sensing purpose. Over the temperature range $140^{\circ} \mathrm{C}$, the pressure and temperature properties have been measured. For 200 Psi full scale pressure sensitivity is $0.30 \mathrm{mV} / \mathrm{Psi}$ from $25^{\circ} \mathrm{C}$ to $140^{\circ} \mathrm{C}$ has been observed with temperature gradient of $-0.35 \mathrm{mV} \rho \mathrm{C}$, with good linearity. The extra resistor which was placed outside the diaphragm acts as a temperature sensor with temperature gradient of about $+0.70 \%{ }^{0} \mathrm{C}^{-1}$.

Keywords: High Pressure, Temperature, MEMS Sensor, Polysilicon, Piezoresistive pressure sensor, Bulk micromachining, Temperature Compensation.
\end{abstract}

\section{Introduction}

After the development of solid state sensors technology, silicon piezoresistive sensors have been widely used, for their advantages of miniaturization, high sensitivity, good linearity, no hysteresis and ability for integration and multifunction. Silicon based piezoresistive pressure sensor have been widely used for various applications ranging from automotive to bioelectronics. For measuring both temperature and pressure simultaneously, different ways are followed and researches are going on. Generally to compensate for the temperature effect, a p-n junction based temperature sensor is often integrated with the silicon pressure sensor. A high temperature pressure and temperature multifunction sensor has been reported [12]. However there is no recent report on MEMS based high pressure and temperature multifunction sensor.

But this high Pressure and moderate temperature MEMS based multifunction sensor can compensate the pressure signal precisely in full temperature range by means of exact temperature signal of the pressure output. In addition, the detection of both to pressure and temperature extends the function of the sensors so that the size and cost of the sensors system can be reduced.

\section{Sensors structure and fabrication:}

A piezoresistive polysilicon high pressure sensor and thermometer of polysilicon resistor are integrated on the mono-chip of the sensor. The structure, design and fabrication of the high pressure and temperature multifunction sensor are introduced as follows:

\subsection{High pressure polysilicon pressure sensor design:}

A Piezoresistive pressure sensor consists of two major active components namely, diaphragm and piezoresistors. The piezoresistors are placed on the diaphragm in configuration of Wheatstone bridge. The change in the resistances due to strain on the diaphragm develops potential difference, which translate the applied pressure. In order to increase the sensitivity of the bridge, the resistances are placed on the diaphragm so as to get maximum change in the potential difference. The piezoresistors change their values in contrast while interacting with tensile and compressive region of stress. Therefore two of the resistors of the bridges are placed in the region of one type of stress while remaining two, are placed in the region of opposite type of stress. The shapes of the resistors were chosen in order to ensure the required change due to diaphragm strain. Dimensions of the resistors were set uniformly of width 10 microns and length 400 microns each. On the basis of the polysilicon layer is estimated for a given value of resistance of four Kilo Ohms. The sheet resistivity of the polysilicon layer is needed for required doping of the layer with suitable specie. The necessary design methodology can be found in the following:

The resistance is mathematically related to physical parameters of the resistor as shown as - 


$$
R=\rho \frac{L}{A}=\rho \frac{L}{W \times d}
$$

Where $\mathrm{R}$ is the resistance, $\rho$ is the resistivity and $\mathrm{L}, \mathrm{W}$ and $\mathrm{d}$ are the length, width and thickness of the resistors.

$$
\begin{aligned}
R & =\frac{\rho}{d} \times \frac{L}{W} \\
\rho_{s} & =\frac{R \times W}{L}
\end{aligned}
$$

Where $\rho_{s}$ is the sheet resistivity.

A diaphragm of square in shape with $2 \mathrm{~mm}$ side and thickness 50 micron was considered to meet the specific requirements in this work. The overall chip shape was set to a square in shape with sides of $4 \mathrm{~mm}$.

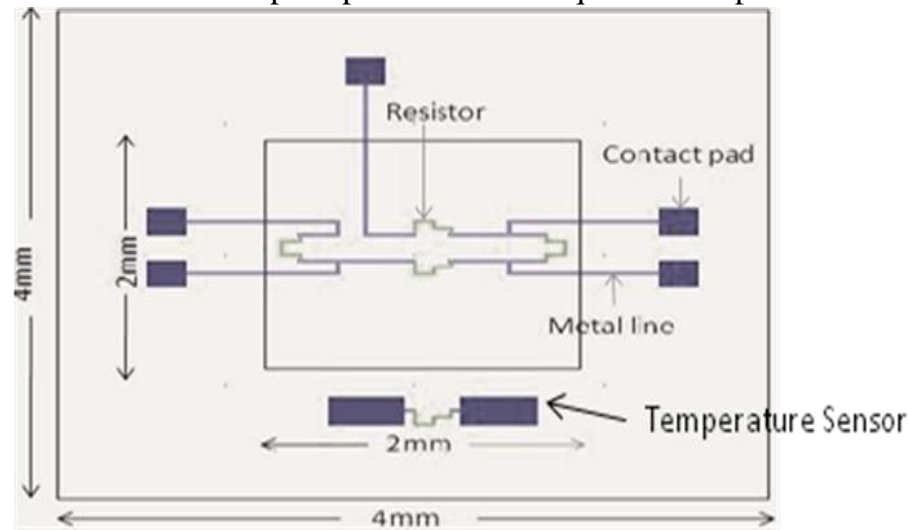

Fig1: The schematic details of bridge over the diaphragm

Summary of major device design parameters:-

Chip size $\quad: 4 \mathrm{~mm} \times 4 \mathrm{~mm}$

Diaphragm size $: 2 \mathrm{~mm} \times 2 \mathrm{~mm}$

Resistor's Value : $2 \mathrm{~K} \Omega$

Resistor's line length $\quad: 400 \mu \mathrm{m}$

Resistor's line width $\quad: 10 \mu \mathrm{m}$

Contact pad locations $\quad: 250 \mu \mathrm{m}$ away from the diaphragm edge

Contact pad Size : $200 \mu \mathrm{m} \times 200 \mu \mathrm{m}$

Metal line width : $20 \mu \mathrm{m}$

Diaphragm thickness $\quad: 50 \mu \mathrm{m}$ (after MEMS process)

\subsection{Temperature sensor design:}

From Fig:1 it can be seen that in the pressure non sensitive area on the chip ,another piezoresistor is integrated as a temperature sensor. As this resistor is situated out of the diaphragm there is no pressure effect on this resistor but the value of the resistor increases with temperature. The dimension of this resistance is also same i.e.

Resistor's line length: 400 micron

Resistor's width : 10 micron

Resistor's value : : 4 kilo Ohms

This resistor is also made by the same process without any additional process.

\section{Fabrication:}

A batch of six wafers of (100) orientation and of $2^{\prime \prime}$ diameter with n-type polarity and both side polished are given chemical cleaning treatment involving degreasing, RCA and piranha. Then the wafers are loaded in the furnace for oxidation for a thickness of 5000^. The oxided wafers are loaded in the reactor for silicon nitride deposition using LPCVD at $780^{\circ} \mathrm{C}$ and 0.3 torr pressure. A thickness of $1500 \AA$ is deposited. In the LPCVD process deposition takes place on both the surfaces of the silicon wafers, which is also good for overall up keep of the wafers during $\mathrm{KOH}$ treatment.

First photolithography is carried out for delineation of holes on the edges of the wafers. Silicon Nitride is selectively etched using RIE(Reactive Ion Etching), thereafter $\mathrm{SiO}_{2}$ is wet etched so that silicon surface is left without dielectric. Wafers are then immersed into aqueous $\mathrm{KOH} \mathrm{(45 \% )} \mathrm{as} \mathrm{to} \mathrm{etch} \mathrm{silicon} \mathrm{at} \mathrm{the} \mathrm{locations} \mathrm{of} \mathrm{hole}$ patterns till through holes are emerged on the other side of the wafers. Next photolithography is carried out for 
delineation of grids with alignment marks on both the surfaces of the wafers. Partial etching of silicon nitride is done by RIE so as to make grid pattern and alignment mark on the wafers. One of the surfaces is cavity patterned. The end point detection for required diaphragm thickness is achieved by the formation of cone in the hole specially provided for this purpose.

The wafers are thoroughly cleaned in DI water followed by HCL treatment for the neutralization of $\mathrm{KOH}$ traces. DI water rinse is given again prior to backing the wafers at $250^{\circ} \mathrm{C}$ for two hours. Now the wafers are loaded for LPCVD polysilicon deposition at $300^{\circ} \mathrm{C}$ for a thickness of $5000 \AA$ on the diaphragm side of the wafers. After this process the wafers are loaded in the furnace for boron doping at $1050^{\circ} \mathrm{C}$ for 45 minutes in $\mathrm{N}_{2}$ environment. The deposited borosilicate is removed by wet oxidation followed by dilute HF dip. Then the sheet resistivity of the polysilicon is measured so as to qualify the required sheet resistivity.

Now photolithography is done for delineation of resistors. Selective etching of polysilicon is done by RIE. Then the wafers are loaded for metallization (blanket) of Al by sputtering for a thickness of 1.0 $\mu$ m.Metal lines and pads are delineated. $\mathrm{Al}$ is selectively etched using following etchant for fine line:

41: 1: $4: 1:: \mathrm{H}_{3} \mathrm{PO}_{4}: \mathrm{HNO}_{3}: \mathrm{CH}_{3} \mathrm{OOH}: \mathrm{H}_{2} \mathrm{O}$

The wafers are loaded in the furnace for sintering at $450^{\circ} \mathrm{C}$ for 30 minutes in forming gas environment. On chip characterization of the resistors are carried out at this stage. Wafers are loaded for PECVD $\mathrm{Si}_{3} \mathrm{~N}_{4}$ deposition. Pads are opened using photolithography and PECVD $\mathrm{Si}_{3} \mathrm{~N}_{4}$ is selectively etched by RIE. Then wafers are ready for chip level testing.

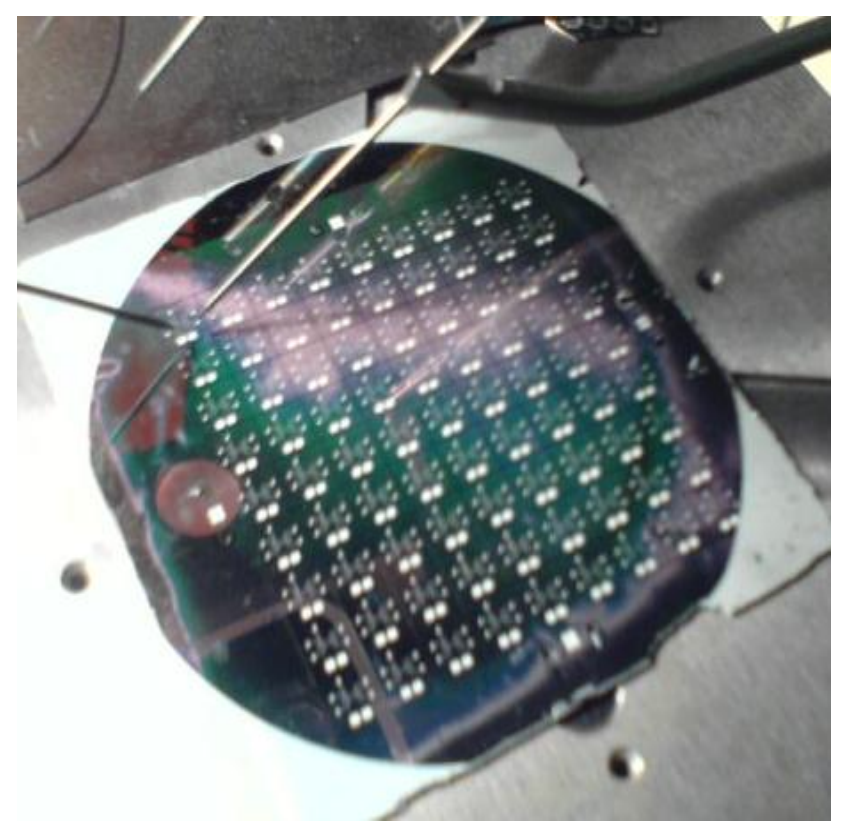

Fig2: Photograph of the processed wafer with 9x9 array of devices

\section{Characteristics of sensors:}

To test the pressure and temperature sensor in actual environment a suitable arrangement is made to apply pressure over the diaphragm and temperature on the chip, which is hermetically sealed over a header using a cap. The pressure and temperature properties of the sensors have been measured by means of this self made pressure and temperature testing system as shown in the fig 3 .

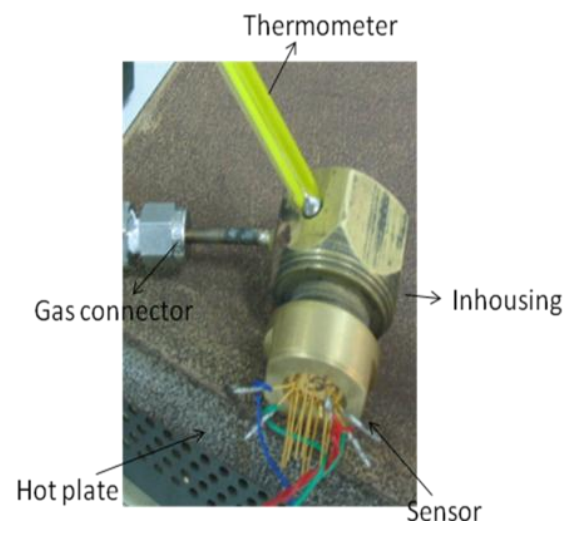


Fig3: Photograph of the multifunction sensor in packaged form

From room temperature $25^{\circ} \mathrm{C}$ to $140^{\circ} \mathrm{C}$ the sensor was tested for full scale pressure of $200 \mathrm{psi}$ and got the following results. The fig. 4 shows the pressure output response of the sensor till 200psi at different constant temperature in the temperature range $25^{\circ} \mathrm{C}$ to $140^{\circ} \mathrm{C}$.

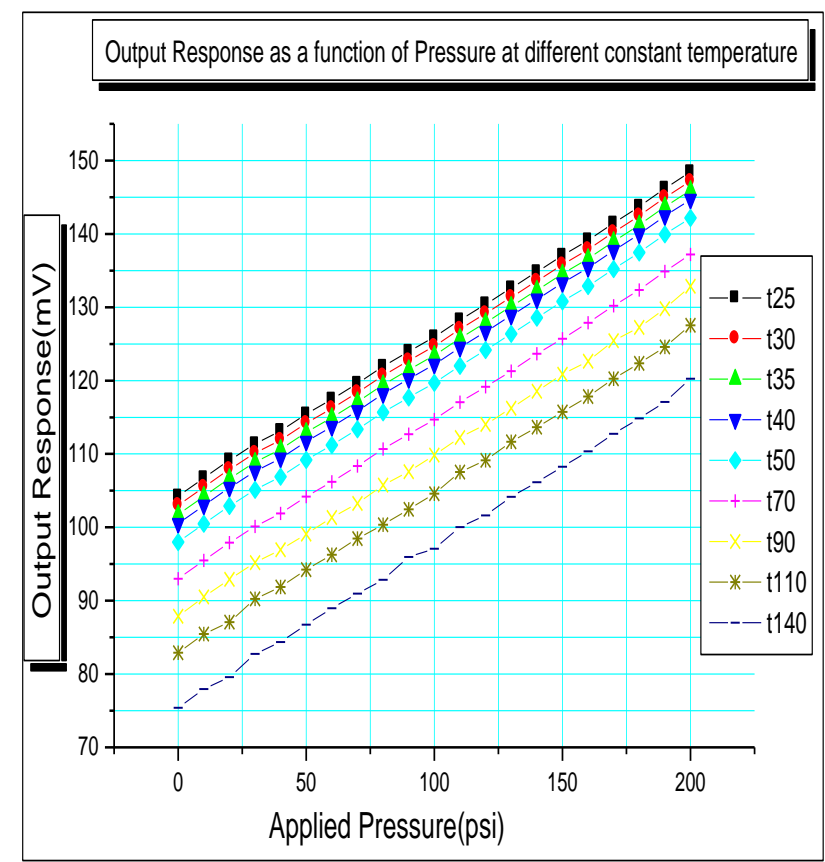

Fig 4: Output Response as a function of applied pressure at different constant Temperature

The full scale pressure output voltage change is $43.4 \mathrm{mV}$ with bridge bias voltage 5V. Also this results shows that there is no temperature effect on the pressure sensitivity of the sensor output from 25 to $140^{\circ} \mathrm{C}$. The typical pressure sensitivity of the sensor is $0.30 \mathrm{mV} / \mathrm{psi}$ with temperature gradient $-0.35 \mathrm{mV} / \mathrm{C}$. The sensor was also tested with varying temperature at different constant pressure and got the results as shown in the Fig: 5.

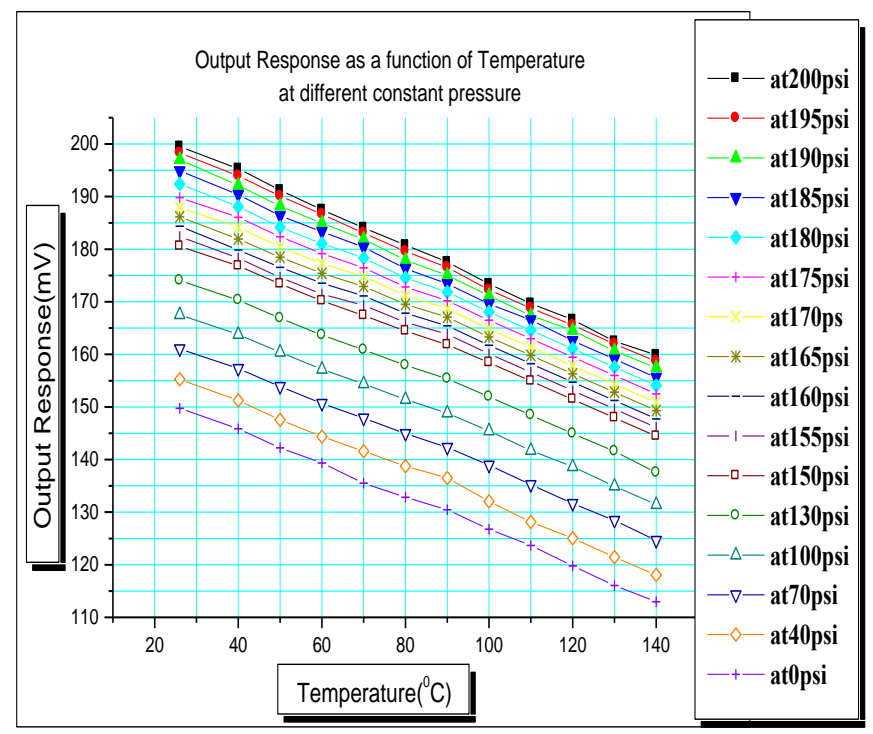

Fig 5: Output response as a function of varying temperature at different constant

pressure. 
These results show that the pressure output of the sensor decreases linearly with temperature and increases with applied pressure.

From the above results it can be estimated that the output voltage of the sensor follows the following equation till $140^{\circ} \mathrm{C}$ :

$$
V_{\text {out }}=T_{s} \Delta T-P_{s} \Delta P
$$

Where $T_{s}=$ Temperature sensitivity of the sensor, $P_{s}=$ Pressure sensitivity of the sensor, $\Delta T$ is the change in temperature, $\Delta \mathrm{P}$ is the change in pressure.

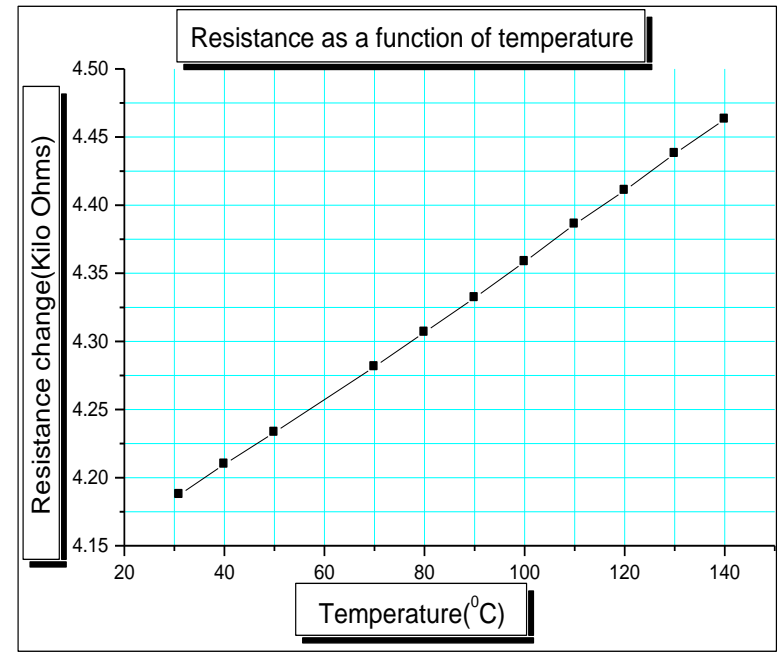

Fig 6: Resistance as a function of temperature rise

Fig 6: shows the temperature sensitive property of the sensor. The extra resistor which is placed out of the diaphragm increases with the temperature rise with good linearity. We also made a wheatstone bridge circuit on bread broad and took this resistance as one of the arm of the wheatstone bridge circuit and found that the temperature sensitivity of this bridge is about $+0.56 \mathrm{mV} /{ }^{\circ} \mathrm{C}$.

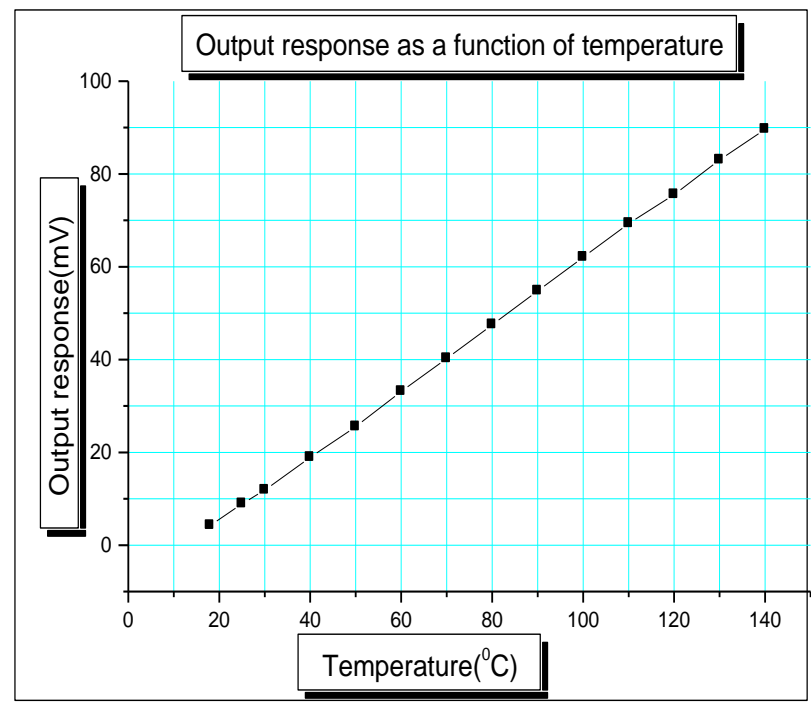

Fig 7: Output response as a function of temperature of the temperature sensor

\section{Conclusion:}

This multifunction sensor was tested to $140^{\circ} \mathrm{C}$ and pressure range of $200 \mathrm{psi}$. The temperature output of the extra resistor has good linearity. The sensor's pressure output also decreases linearly with temperature rise. So, it is easy to compensate the temperature effect of the pressure output as there is no temperature effect on the sensitivity of the pressure output. Furthermore because the temperature sensor and pressure sensor are integrated on the same chip, the temperature output is the exact temperature at the detected pressure position. This can 
realize the precise compensation of the temperature offset of the pressure output of the sensors in the full temperature range.

\section{References:}

[1]. J. Akhtar "Polysilicon Piezoresistive Pressure Sensors based on MEMS Technology" , IETE Journal of Research, Vol no. 49, 2003, pp 365-377.

[2]. Jorge Ramirez and Edgar Charry, “A CMOS signal conditioning circuit for piezoresistive pressure sensors”, IEEE2002, ISBN: 07803-7448-7, pp592-595.

[3]. Wu S, Mai J, Zohar Y, Tai Y C and Ho C M, "A suspended micro-channel with integrated temperature sensor of high pressure flow studies”,1997-1998,http://ho.seas.ucla.edu/publications/\#c97

[4]. C Pramanik, H Saha and U Gangopadhyay, "An integrated pressure and temperature sensor based on nanocrystalline porous silicon", Journal of Micromechanics and Microengineering, Vol.16, 2006, pp 1340-1348

[5]. Moon key Lee, Bo Na Lee and Young Jun Lee," A signal conditioning circuit for silicon pressure sensor", IEEE1992, ISBN: CH3177-3, pp250-253.

[6]. Islam Baharul, Sharma Pavika, Das Surajit, Akhtar Jamil, ”A digital pressure meter based on piezoresistive polysilicon MEMS sensor", journal of instrument society of India, vol.40, no.1, pp36-38.

[7]. Pavika Sharma, Baharul Islam and J. Akhtar, "Characterization and response analysis of a polysilicon piezoresistive MEMS sensor for pressure and temperature", under process in Sensors and Actuators A Physical, Elsevier.

[8]. Robert Bogue, "MEMS sensors-past, present and future", Emerald Journal Sensor Review, Volume $27 \cdot$ Number $1 \cdot 2007$.

[9]. Jorge Ramirez and Edgar Charry, "A CMOS signal conditioning circuit for piezoresistive pressure sensor" IEEE, 2002.

[10]. Pavika Sharma, Prerna Garg, and P. A. Alvi, "Designing of Water Quality Detector Using pH Sensor", Sensors \& Transducers Journal, Vol. 105, Issue 6, June 2009, pp. 42-49.

[11]. Liu Xiaowei, Wang Wei, Wang Xilian Liu Yuqiang, Liu Zhenmao,"High-temperature Pressure and Temperature Multi-function sensors“, IEEE,1998,0-7803-4306-9/98/pp 947-949.

[12]. Shilie Zheng and Xianmin Zhang, "Simulataneous measurement of pressure and temperature using a single fibre Bragg Grating", Progress in electromagnetic research symposium,2005, Hangzhou, China, August 22-26. 